\title{
Liga 55\% Al-Zn: uma alternativa anticorrosiva para o setor industrial
}

\section{Alloy 55\% Al-Zn: An anti-corrosive alternative for the industrial sector}

\author{
1 Wilma Clemente de Lima Pinto \\ 1 Gleyce Pereira da Silva \\ 1 Mauro Carlos Lopes Souza mauroclsouza@hotmail.com \\ 2 Tania Maria Cavalcante Nogueira
}

1 Centro Universitário Estadual da Zona Oestre - UEZO, RJ.
2 Escola de Engenharia Industrial e Metalúrgica Volta Redonda - UFF, RJ.

\section{Resumo}

Chapas de aço carbono, revestidas com a liga 55\% Al-Zn são usadas pela indústria devido a elevada resistência à corrosão atmosférica, conferindo maior durabilidade ao aço e maior resistência à corrosão por barreira conferida pelo alumínio, combinada à proteção galvânica do zinco. As amostras T1 e T2 foram estudadas por meio da técnica voltamétrica em meio neutro de $\mathrm{KNO}_{3}$, $0,2 \mathrm{M}$, contendo cloreto. Os processos eletroquímicos foram monitorados pela técnica de microscopia eletrônica de varredura. Os resultados de voltametria mostraram uma maior resistência à dissolução para a liga com o tratamento de cromatização $T 1$, sendo que, na presença de cloreto, ocorreu também a dissolução da fase dendrítica rica em alumínio. Após 403 dias de exposição de campo em ambiente marinho, o avanço da corrosão mostrou que, em T1, o zinco foi completamente consumido nos espaços interdendríticos, enquanto, em T2, ocorreu ataque nas dendritas. Após 1272 horas de exposição de T1 e T2 em câmara úmida, T1 apresentou uma área exposta com corrosão branca localizada enquanto T2 teve uma maior densidade de pontos com corrosão branca. No ensaio de névoa salina após o mesmo tempo de exposição, T1 apresentou área exposta totalmente tomada por corrosão branca com alguns pontos de corrosão vermelha e T2, além de apresentar área exposta completamente tomada por corrosão branca mostrou uma maior densidade de pontos com corrosão vermelha. Esses resultados foram coerentes com àqueles encontrados na voltametria, nos quais T1 possui maior resistência à corrosão que T2, provavelmente ocasionado pelo maior conteúdo de cromo total.

\section{Palavras-chave:}

Liga 55 \% Al - Zn. Voltametria. Microscopia Eletrônica. Ensaios Acelerados.

\begin{abstract}
Carbon steel sheets, coated with 55\% Al-Zn alloy, are used by the industry due to their high resistance to atmospheric corrosion, providing greater durability to the steel due to the greater resistance to barrier corrosion provided by aluminum, combined with the galvanic protection of zinc. Samples T1 and T2 were studied using the voltammetric technique in a neutral $\mathrm{KNO}_{3}$ medium, $0.2 \mathrm{M}$ containing chloride and the electrochemical processes were monitored by the technique of scanning electron microscopy. The voltammetry results showed a higher dissolution resistance for the alloy with the T1 chromating treatment, in the presence of chloride, the aluminum-rich dendritic phase also dissolved. After 403 days of field exposure in a marine environment, the advance of corrosion showed that, in T1, zinc was completely consumed in the interdendritic spaces, while in T2 there was an attack on the dendrites. After 1272 hours of exposure of T1 and T2 in a wet layer, T1 showed an exposed area with localized white corrosion while T2 had a higher density of white corrosion spots. In the saline mist test, after the same exposure time, T1 showed an exposed area completely taken up by white corrosion with some spots of red corrosion and T2 in addition to presenting an exposed area completely taken up by white corrosion showed a higher density of spots with red corrosion. These results were consistent with those found in voltammetry, where T1 has greater corrosion resistance than $\mathrm{T2}$, probably caused by the higher total chromium content.
\end{abstract}

\section{Keywords:}

55\% Al-Zn alloy. Voltammetry. Electron Microscopy. Accelerated Tests.

\section{Como você deve citar?}

PINTO, Wilma Clemente de Lima et al. Liga 55\% Al-Zn: uma alternativa anticorrosiva para o setor industrial. Cadernos UniFOA, Volta Redonda (RJ), v. 16, n. 46, p. 1-9, ago, 2021. 


\section{INTRODUÇÃO}

O galvalume é um revestimento aplicado ao aço carbono que consiste em uma liga 55\%Al-Zn. Esse revestimento se caracteriza por uma microestrutura composta de cerca de $80 \%$ em volume da fase a-dendrítica rica em alumínio e cerca de $20 \%$ da fase interdendrítica rica em zinco, conferindo resistência à corrosão devido à proteção por barreira do alumínio combinada à proteção galvânica do zinco (TOWNSEND et al. 1985; PINTO, 2003).

Pesquisas relatam que a corrosão da fase rica em alumínio não ocorrerá até que a fase rica em zinco seja completamente consumida (SARLI et al. 2003).

O Galvalume tem sido submetido às atmosferas, industrial, rural e marinha (moderado e severo). A principal diferença entre estes ambientes reside na presença ou na ausência de íons cloreto (HARVEY, 1981).

$\mathrm{Na}$ atmosfera industrial e/ou rural, o Galvalume comporta-se mais como uma camada de alumínio com proteção nas bordas, sendo fornecida pela ação galvânica da fase rica em zinco. Essa fase age como uma fonte de íons zinco, que atua como um inibidor; e resulta na formação de produtos de proteção à corrosão (DALLEDONE; BARBOSA; WOLLYNEC, 1995).

Em ambiente marinho, toda a camada fornece proteção galvânica, auxiliada pela quebra da passividade do filme de óxido, devido à maior quantidade de íons cloreto presentes. Portanto, existe proteção catódica a partir de ambas as fases principais na camada. Nesse ambiente, ocorre também uma dissolução mais rápida da fase rica em zinco, comparando-se a ambientes menos agressivos.

Para melhorar a resistência à corrosão da liga $55 \%$ Al-Zn é necessário depositar, em sua superfície, uma camada orgânica de compostos insolúveis de cromo, fosfatos e resina orgânica. Os estudos comprovaram um excelente resultado para fosfatos, pois um filme de proteção (camada passivada) é formado, inibindo o mecanismo corrosivo (TOWNSEND; BORZILLO, 1996; YAMASHITA; YAMAJI; MATSUZAKI., 2003).

A liga 55\% Al-Zn tem sido usada para proteger aço carbono da corrosão atmosférica, entretanto, no caso de armazenamento em ambientes úmidos, para aumentar a resistência à corrosão do revestimento evitando o manchamento e preservando o aspecto superficial do aço é realizado um tratamento de superfície à base de cromato, fosfato ou resina acrílica após a obtenção da liga. Os tratamentos à base de cromatos têm sido os mais indicados ao longo do tempo, tanto para proteção temporária (passivação) quanto como pré-tratamento para pintura (MORCILLIO, PALMA; PUENTE, 1998).

De acordo com HAGANS et al, 1994 e HORNSTROM et al, 1993 o tratamento de cromatização, além de prevenir a corrosão temporária, é também substratos necessários para a boa adesão em caso de pintura. Uma outra aplicação é decorativa, pois esse tratamento confere cor e brilho ao substrato

Os estudos preliminares para conhecer a resistência à corrosão atmosférica do galvalume com diferentes composições de alumínio e zinco se iniciaram na década de $60 \mathrm{com}$ a Cia de aço Bethlehem. Desde essa data o galvalume vem sendo submetido, periodicamente a ensaios de resistência à corrosão. Encontram-se estudos relatando a eficiência da liga à exposição atmosférica após 13 anos (TOWNSEND; ZOCCOLA, 1979), 20 anos (TOWNSEND e BORZILLO,1987), 25 anos (TOWNSEND; BORZILLO, 1993) e; 30 anos (TOWNSEND; BORZILLO,1996 e PINTO, 2003). Todos confirmaram que a camada 55\%Al-Zn sobre aço carbono fornece uma ótima combinação de durabilidade, proteção sacrificial e resistência à corrosão em todos os ambientes, sendo esta proteção uma consequência da microestrutura da liga e do mecanismo de corrosão 
Ensaios eletroquímicos são importantes nos estudos dos mecanismos de proteção. Essas técnicas também são baseadas em ensaios acelerados e decorrência do contato contínuo da amostra com o eletrólito. A aplicação de técnicas eletroquímicas ao estudo de depósitos têm sido de grande importância no caso de ligas depositadas, pois permite obter informações com relação à composição das fases presentes, bem como o mecanismo de atuação da liga (CARLOS, 1990; BONFÁ et al,1991).

A voltametria tem sido usada como uma técnica eletroanalítica de superfície (CARLOS, 1992). Essa técnica tem ampla aplicação no conhecimento das propriedades da superfície, entre elas, sua composição.

O processo de dissolução da liga cromatizada tem sido amplamente estudado utilizando-se a técnica de voltametria cíclica, que produziu resultados que foram concordantes com a caracterização microestrutural por microscopia eletrônica de varredura (WALLINDER et al,1991, NOGUEIRA, 1999, 2000, 2001; PINTO, 2003).

Embora a utilização da liga 55\% Al-Zn tenha sido estudada desde a década de 60, devido ao grande interesse comercial, o estudo da microestrutura relacionada ao processo de corrosão continua motivando o interesse de pesquisadores em comprovar o seu uso como uma alternativa para o setor industrial.

O objetivo deste trabalho é estudar da liga cromatizada e camada de cromatização, utilizando-se amostras com dois tratamentos diferentes de cromatização. Pretende-se correlacionar os dados obtidos desses estudos com as características de propriedades anticorrosivas e o substrato cromatizado. bem como a monitoração da superfície das amostras. Técnicas de ensaios acelerados, eletroquímicas e de análise de superfície que permitem uma avaliação da eficiência do tratamento proposto, bem como da resistência à corrosão também são analisadas.

\section{MATERIAIS E MÉTODOS}

\subsection{Materiais}

Foram utilizadas amostras de aço carbono revestidas com a liga 55\% Al-Zn:

T1 - As amostras T1 foram utilizadas como recebidas da importação da Austrália pela empresa Tekno. Consiste de chapas de aço carbono recobertas com a com a liga 55\%Al-Zn, submetidas a um tratamento prévio de conversão de camada por cromatização, em ambas as faces.

T2 - Amostras T1 que foram submetidas a um segundo tratamento de cromatização, na empresa Tekno. Esse segundo tratamento tem como objetivo aumentar a rugosidade do material. 0 banho utilizado consistiu de uma mistura de ácido crômico e aço fosfórico contendo compostos de fluoreto, aplicado pelo processo Roll on.

\subsection{Métodos}

\subsubsection{Determinação da massa de cobertura padrão}

As amostras T1 e T2 foram cortadas na dimensão 2,0 x 2,0 cm, pesadas em balança analítica de precisão ao décimo do miligrama e, em seguida foram imersas por um minuto em trinta mililitros de solução decapante de ácido clorídrico na proporção $1: 1$, à temperatura de $22^{\circ} \mathrm{C}$, contendo o inibidor de corrosão hexametileno tetramina. Posteriormente foram lavadas, secas e novamente pesadas. A diferença de peso e a área das amostras foram utilizadas para o cálculo do peso do revestimento em $\mathrm{g} / \mathrm{cm}^{2}$. 


\subsubsection{Determinação de cromo total}

Da solução resultante da decapagem ácida, foi determinada a concentração de cromo total por espectrometria de absorção atômica, usando-se um espectrômetro de absorção atômica marca Varian, modelo espectra A 800. A partir dos padrões de concentração de 1000ppm (Merck) do elemento cromo, foram elaboradas curvas de calibração. As medidas foram realizadas em triplicata à temperatura de $22^{\circ} \mathrm{C}$.

\subsubsection{Ensaios Voltamétricos}

As amostras foram submetidas ao ensaio de voltametria cíclica, utilizando-se o potenciostato FAC- 200A, um gerador de rampa FAC- 200B e um registrador marca Yokogawa Hokushin Electric modelo $X-Y$ 3036. Foi utilizada uma célula convencional a três elétrodos, onde os elétrodos de trabalho foram as amostras $\mathrm{T} 1 \mathrm{e}$ T2, previamente desengordurados com acetona e secos ao ar; o contra eletrodo, um fio de platina e o eletrodo de calomelano em $\mathrm{KCl} 1 \mathrm{M}$ foram utilizados como referência. 0 ensaio foi realizado a partir do potencial inicial de $-1,3 \vee$ e, a varredura inicial anódica teve o seu sentido invertido em torno de 1,0 V, terminando no mesmo potencial inicial, tendo como eletrólito a solução de nitrato de potássio $0,2 \mathrm{M}$ em cloreto de sódio $0,01 \mathrm{M}$. As análises foram realizadas à temperatura ambiente com aeração natural; a velocidade de varredura foi de $10 \mathrm{mV} \cdot \mathrm{s}^{-1}$; e a área útil das amostras foi de $9,0 \mathrm{~cm}^{2}$.

\subsubsection{Exposição de campo em estação de corrosão atmosférica marinha}

A avaliação da resistência à corrosão atmosférica das amostras T1 e T2 foi realizada através de ensaio de campo em exposição atmosfera marinha (estação de corrosão atmosférica do CEPEL). Com a finalidade de acelerar o ensaio, as amostras foram borrifadas com solução de $\mathrm{NaCl} 1 \%$ duas vezes por semana durante o período de 403 dias. A estação de teste utilizada foi montada seguindo-se a norma ISO 9223 e corpos de prova foram de dimensão $10 x 15 \mathrm{~cm}$. Após o período de exposição, as amostras T1 e T2 foram avaliadas quanto ao avanço de corrosão por microscopia eletrônica de varredura.

\subsubsection{Análise da seção transversal}

Após o período de 403 dias de exposição das amostras à atmosfera marinha, as amostras foram cortadas e embutidas em baquelite. A micrografia da seção transversal de T1 e T2 foi realizada utilizando-se o microscópio eletrônico de varredura (MEV) Zeiss modelo DSM-962, com aumento de 2000X.

\subsubsection{Ensaio de Névoa Salina}

As amostrasT1 e T2 foram preparadas conforme a norma ABNT- NBR 8094, com dimensão de $10 \times 15 \mathrm{~cm}$. Uma solução de cloreto de sódio $5 \%$ foi borrifada por um spray dentro de uma câmara à temperatura de $35^{\circ} \mathrm{C}$ e pressão entre $70 \mathrm{KPa}$ e $170 \mathrm{KPa}$, por 1272 horas. 0 ensaio foi realizado em triplicata e, após o período de exposição, os corpos de prova foram analisados visualmente e fotografados utilizando-se uma câmera digital Sony Cyber Shot.

\subsubsection{Câmara de Umidade}

Amostras T1 e T2 foram preparadas de acordo com a norma ABNT- NBR 8095 (2015), com dimensão de $10 \times 15 \mathrm{~cm}$ e colocadas em uma câmara com umidade relativa de $100 \%$ durante 1272 horas e submetidas à condensação constante de umidade durante todo o ensaio, também realizado em triplicata. Após o período de exposição, os corpos de prova foram analisados quanto ao aspecto visual e fotografados com uma câmera digital Sony Cyber Shot. 


\section{RESULTADOS E DISCUSSÃO}

Os resultados de peso de revestimento obtidos para as amostras T1 e T2 foram 163,2 g/m² e $160,6 \mathrm{~g} / \mathrm{m}^{2}$ respectivamente.

Os resultados de peso de cromo total obtidos para as amostras T1 e T2 foram, respectivamente, $24,93 \mathrm{mg} / \mathrm{cm}^{2}$ e $16,54 \mathrm{mg} / \mathrm{cm}^{2}$. De acordo com esses resultados após o tratamento de conversão de camada há perda de cromo no filme.

A Figura 1 refere-se ao comportamento voltamétrico das amostras T1 e T2 na solução $\mathrm{NaCl} 0,01$ $\mathrm{M}$ e $\mathrm{KNO}_{3}$ 0,2 $\mathrm{M}$. As densidades de carga para as amostras T1 e T2 são $20,2 \pm 2,0 \mathrm{mC} / \mathrm{cm}^{2}$ e 41,2 $\pm 2,2$ $\mathrm{mC} / \mathrm{cm}^{2}$, respectivamente.

Pode-se ressaltar que a densidade de carga anódica do voltamograma pode ser atribuída tanto à dissolução do zinco quanto à do alumínio. Observou-se que a amostra T1 apresenta menor dissolução que a amostra T2. A presença de um pico anódico na varredura catódica mostra um processo de reativação que só pode corresponder à dissolução do zinco interdendrítico (PINTO, 2003). Um outro resultado importante se relaciona com as densidades de corrente anódica para as duas amostras. Para a amostra com menor teor de cromo no filme (T2) as densidades de corrente são maiores. Todos esses resultados mostram a influência do filme de cromatização desfavorecendo o processo de dissolução do zinco na liga.

Figura 1 - Voltametria de T1 e T2 em NaCl 0,01 M + $\mathrm{KNO}_{3} 0,20 \mathrm{M}\left(10 \mathrm{mV} \mathrm{s}^{-1}\right)$

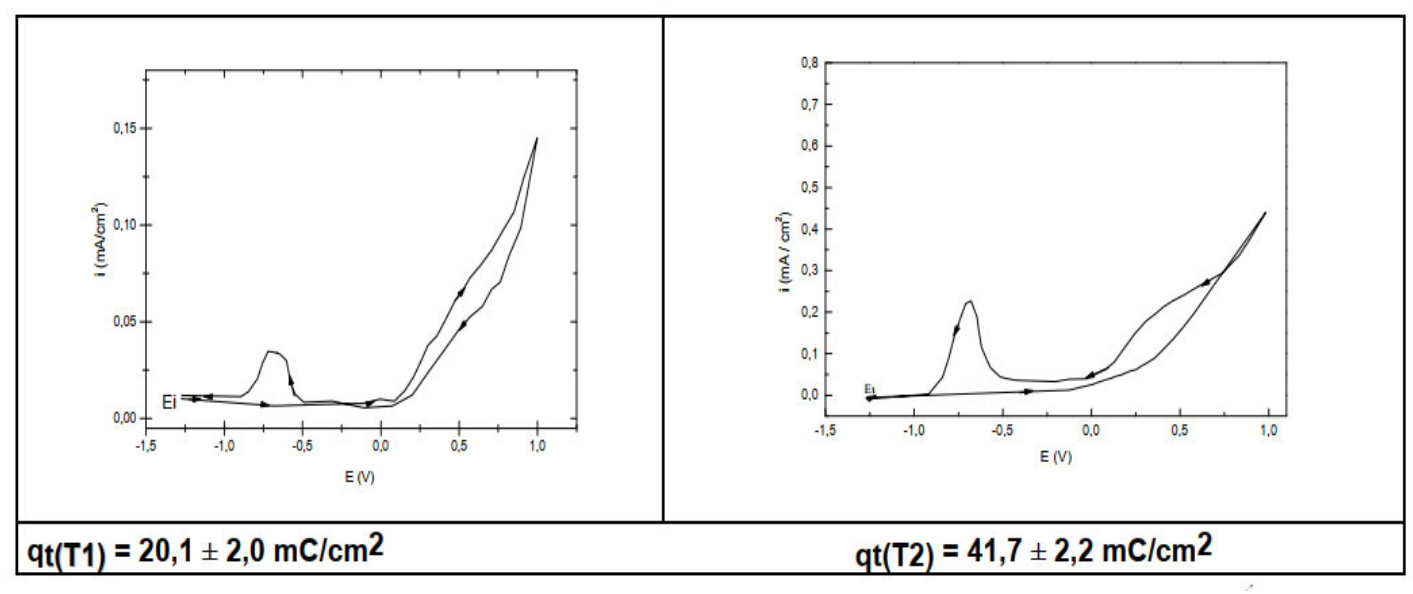

Fonte: Dos autores

Para verificar se os dados voltamétricos têm de fato correlação com o desempenho frente à resistência à corrosão, T1 e T2 foram paralelamente avaliadas quanto ao processo corrosivo. Para tanto as amostras foram expostas em ensaio de campo em ambiente marinho por um período de 403 dias. Ao final da exposição, foram avaliadas quanto ao avanço de corrosão por MEV.

Foi observado o avanço da corrosão em T1 e T2 após a exposição de campo, ao ambiente marinho, utilizando-se MEV de seção transversal no final de 403 dias. As micrografias são mostradas na Figura 2. Observa-se que o zinco foi completamente consumido nos espaços interdendríticos, evidenciando um ataque muito maior nessas regiões em relação à amostra $\mathrm{T} 1$. Na amostra $\mathrm{T} 2$ pode ser verificado também um ataque nas dendritas, o que não é evidenciado na amostra T1. 0 avanço de corrosão observado em T1 e T2 está de acordo com outras pesquisas (HARVEY, 1981, TOWNSEND, BORZILLO, 1996). Neste 
trabalho os autores estudaram o mecanismo de corrosão através da observação da microestrutura da camada da liga e concluíram que a fase rica em zinco, presente em menor proporção na microestrutura, corrói preferencialmente até que a formação de produtos de corrosão cessa qualquer reação posterior na área. Quando a fase rica em zinco se extingue, a velocidade de corrosão torna-se mais característica da fase rica em alumínio, a qual está presente em maior proporção na camada, resultando em uma menor velocidade de corrosão, típica do alumínio.

Figura 2 - Micrografia da seção transversal de T1 e T2 com aumento de 2000x após 403 dias de exposição em ambiente marinho.

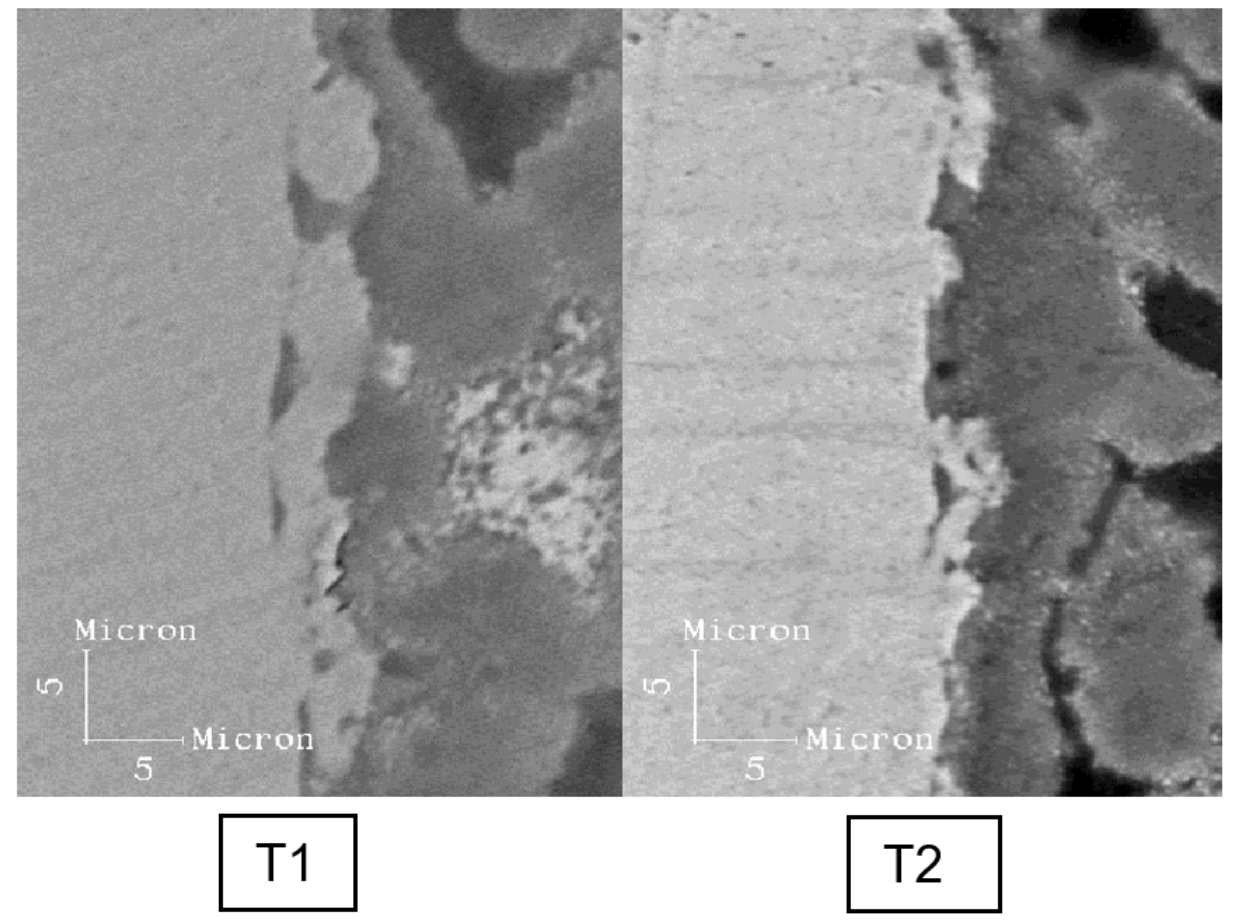

Fonte: Dos autores

A figura 3 mostra os resultados dos ensaios acelerados de laboratório tais como, névoa salina e câmara úmida por 1272 horas. Observa-se que no ensaio de câmara úmida, T1 apresenta uma área exposta com corrosão branca localizada enquanto T2 tem uma maior densidade de pontos com corrosão branca.

No ensaio de névoa salina observa-se que T1 possui área exposta totalmente tomada por corrosão branca, com alguns pontos de corrosão vermelha, e T2, além de apresentar área exposta completamente tomada por corrosão branca mostra uma maior densidade de pontos com corrosão vermelha.

Cabe ressaltar que tanto T1 e T2, após 504 horas de ensaio de névoa salina, já apresentavam corrosão branca, enquanto no ensaio de câmara úmida observou-se o primeiro sinal de corrosão a aproximadamente, 884 horas. 
Figura 3 - Fotografias de T1 e T2 submetidos aos ensaios de névoa salina e câmara úmida por 1272 horas.

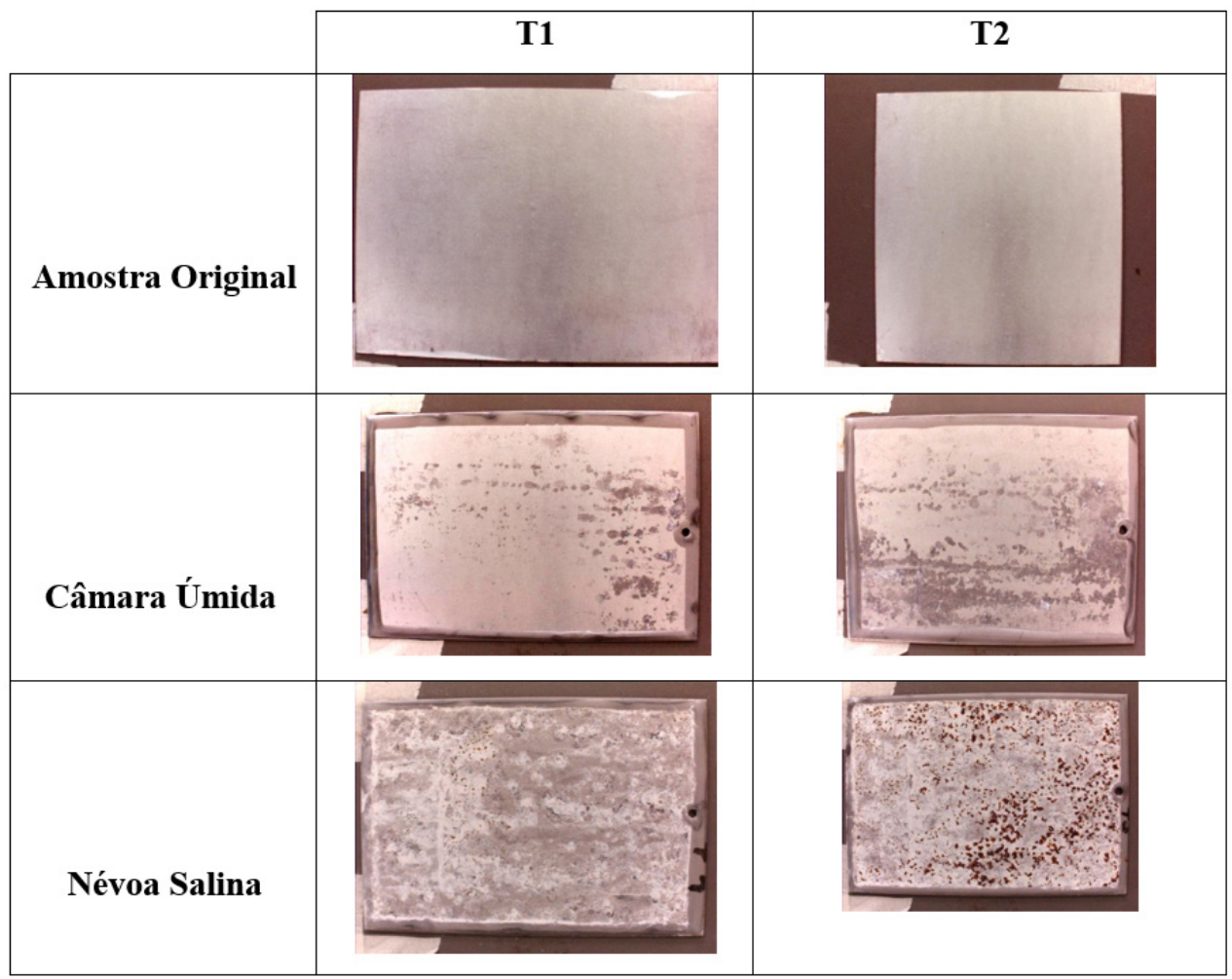

Fonte: Dos autores

Outras pesquisas de Seré et al. (1998), mostraram o comportamento de corrosão da liga 55\% AlZn em exposição aos ensaios acelerados de câmara úmida e névoa salina até 360 horas, semelhantes aos encontrados neste trabalho, entretanto, utilizando um tempo de ensaio consideravelmente menor.

Os resultados dos ensaios de campo e dos ensaios acelerados de laboratório estão concor-dantes com aqueles encontrados na voltametria, nos quais $T 1$ possui maior resistência à corrosão que $T 2$, provavelmente ocasionado pelo maior conteúdo de cromo total.

\section{CONCLUSÕES}

A conversão de camada realizada em amostra da liga 55\% Al-Zn, previamente passivada, apresentou diminuição do teor de cromo total no filme, favorecendo o processo de dissolução do zinco nos espaços interdendríticos.

A camada 55\%Al-Zn sobre aço carbono fornece uma ótima combinação de durabilidade, proteção sacrificial e resistência à corrosão em todos os ambientes, confirmando a possibilidade do uso da liga (55\%Al-Zn) ser utilizada na área industrial.

A voltametria completa em solução neutra não garante a dissolução total do zinco nos espaços interdendriticos. Tanto o filme de cromatização quanto a microestrutura exercem uma forte influência na dissolução voltamétrica do zinco, sendo que esses aspectos não podem ser desprezados na interpretação das curvas voltamétricas. 
A técnica voltamétrica mostrou-se mais eficiente no estudo de dissolução do zinco inter- dendrítico, que a microscopia da seção transversal.

\section{AGRADECIMENTOS}

Os autores agradecem ao suporte financeiro oferecido pela Fundação de Apoio à Pesquisa do Estado do Rio de Janeiro - FAPERJ, ao presente estudo. Agradecemos também ao técnico Fernando dos Reis Franco pelas análises realizadas no MEV, e ao técnico Muricy Ribeiro Brito pela análise de cromo.

\section{REFERÊNCIAS}

ASSOCIAÇÃO BRASILEIRA DE NORMAS TÉCNICAS. ABNT NBR 8094: Material metálico, revestido e não revestido - Corrosão por exposição à névoa salina - Método de Ensaio. Rio de Janeiro: ABNT, 1983.

ASSOCIAÇÃO BRASILEIRA DE NORMAS TÉCNICAS. ABNT NBR 8095. Material metálico, revestido e não revestido - Corrosão por exposição à atmosfera úmida saturada - Método de Ensaio. Rio de Janeiro: ABNT, 2015.

BONFÁ R. M. 1991. Estudo de eletrodepositos de cupro-níquel por técnicas de dissolução eletroquímica. Dissertação Doutorado. São Carlos - SP: UFSCAR/IFQSCOS, 1991 210p.

CARLOS, I.A. 1990. Eletroanalítica de superficies sólidas. dissertação doutorado. São Carlos - SP: UFSCAR/IFQSC, 1990, 200p.

CARLOS, I.A., D`ALKAINE, C.V. 1992. Eletrochemical characterization of Cu/Zn alloy electrodeposits. In: International congress for surface finishing 92, São Paulo, SP, Brasil, Anais. p.1428-1433.

DALLEDONE, E. BARBOSA, M.A., WOLLYNEC, S., 1995. Zinc 55\% Aluminium-1,6\% silicon coating compared with zinc coating, Materiais Performance, v.34, n 7, p. 24-28.

HAGANS, P.L., HAAS, C.M., 1994. Chromate conversion coatings. Asm Internation handbook, v.5, Surface engineering, p.405-411.

HARVEY, G.J., 1981. Structure and corrosion resistance of zincalume coatings. BHP technical bulletin v. 25, n.2, p. $63-67$.

HORNSTROM, S.E.; HELDLUND, E. G.; KLANG, H.; NILSSON, J.O.; BACKLUND, M. ,1993. Surface study of a chromate pretreatment before coil coating of Al-43.4Zn-1.6 Si. Coated steel, Surface and interface analysis, v-20, p.427-433.

NOGUEIRA, T.M.C.; SEIXAS, U.R.; RIOS, P.R., 1999. Aplication of a direct current anodic voltametric technique to a 55\% Al-Zn coated steel before and after an annealing heat. ISIJ international, vol.39 (3), p.295-297.

NOGUEIRA, T.M.C.; CRUZ, M.A.S.; RIOS, P.R., 2000. Electrochemical behaviour of a chromated 55\% Al-Zn coated steel sheet before and after heat treatment, ISIJ international, vol. 40 (8), p.824-826.

MORCILLO, M., PALMA E., PUENTE, J.M., 1998. The atmospheric corrosion mechanism of 55\% Al-Zn coating on steel, Corrosion science, vol 40, No.1, p.61- 68. 
PINTO, W.C.,2003. Estudo da Influência do pré-tratamento da cromatização da liga $55 \% \mathrm{Al}-\mathrm{Zn}$ sobre aço carbono. Tese de Doutorado. Rio de Janeiro - RJ: UFRJ/IQINORG, 2003. p.164.

SARLI, A.R. DI, ALVAREZ, N. B, SACCO, E.A., CULCASI, J. D., ELSNER, C.I., 2003.

Effect of the plastic deformation on the electrochemical behavior of metal coated steel sheets. Surface and coatings technology, v.168, p. 115-112.

SERÉ, P.R., ZAPPONI, M., ELSNER, C. I., 1998. Comparative corrosion behavior of 55 aluminium- zinc alloy and zinc hot-dip coatings deposited on low carbon steel substrates. Corrosion Science, v. 40, n.10 p.1711-1723.

TOWNSEND, H.E, ZOCCOLA, J.C., 1979. Atmospheric corrosion resistance of 55\% Al-Zn coated sheet steel: 13year test results. Materials performance, v. 18, p.13-20.

TOWNSEND, H.E, BORZILLO, A.R., 1987. Twenty-year atmospheric corrosion test of of hot dip coated steel sheet. Materials performance, v. 26 (7), p.37-41.

TOWNSEND, H.E, BORZILLO, A.R., 1993. Twenty-five-year corrosion test of 55\% Al-Zn alloy coated steel sheet. Materials performance, v. 33, p.68-71.

TOWNSEND, H.E, BORZILLO, A.R., 1996. Thirty - year atmospheric corrosion performance of $55 \%$ Aluminium - zinc alloy coated sheet steel. Materials performance, v. 35, p.30-36.

TOWNSEND, H. E., FISHER, T.W., 1985. 55\% Aluminium-Zinc alloy coated steel sheet and wire. Metals Handbook. v. $519^{\text {th }}$ edition.

WALLINDER, O.; HE, W.; AUGUSTSSON, P.E.; LEYGRAF, C., 1999. Characterization of black rust staining of unpassived $55 \% \mathrm{Al}-\mathrm{Zn}$ alloy coating. Effect ot temperature $\mathrm{pH}$ and wet storage. Corrosion science, 41 , p. 2229-2249.

YAMASHITA, M., YAMAJI T., MATSUZAKI, A., 2003. Development of a new organic composite coating for enhancing corrosion resistance of $55 \% \mathrm{Al}-\mathrm{Zn}$ alloy steel sheet, Surface and coatings technology, v. 169-170, p. 655-657. 